\title{
Junior Doctors' Knowledge about Chronic Hepatitis B Guideline: A Survey Among 30 Primary Hospitals in Sichuan Province of China
}

\author{
En-Qiang Chen, ${ }^{1,2}$ Meng-Lan Wang, ${ }^{1,2}$ Lang Bai, ${ }^{1,2}$ Xiao-Fang Lv, ${ }^{1,2}$ Dong-Mei Zhang, ${ }^{1,2}$ Juan Wang, ${ }^{1,2}$ \\ Ya-Chao Tao, ${ }^{1,2}$ and Hong Tang ${ }^{1,2, *}$ \\ ${ }^{1}$ Center of Infectious Diseases, West China Hospital of Sichuan University, Chengdu 610041, PR China \\ ${ }^{2}$ Division of Infectious Diseases, National Key Laboratory of Biotherapy/Collaborative Innovation Center of Biotherapy, West China Hospital of Sichuan University, Chengdu \\ 610041, China \\ "Corresponding author: Hong Tang, Center of Infectious Diseases, West China Hospital of Sichuan University, Chengdu 610041, China, E-mail: htang6198@hotmail.com
}

Received 2016 December 24; Revised 2017 January 20; Accepted 2017 January 28.

\begin{abstract}
Objectives: This study aimed to assess the knowledge of junior doctors on the 2015 chronic hepatitis B (CHB) guideline recommended by Chinese society of infectious diseases and Chinese society of hepatology, Chinese medical association.

Methods: Junior doctors, who were already engaged in the field of $\mathrm{CHB}$, were invited to complete a questionnaire-based survey between May and June 2016. The questionnaire consisted of 28 items focusing on knowledge in the following three sections: motherto-child transmission (MTCT) prevention, response-guided therapy (RGT) strategies, and special patients' antiviral therapy.

Results: Responses were received from 562 out of 600 participants. Among those 562 junior doctors, about 10\% 30\% were not familiar with the use of hepatitis B vaccine and hepatitis B immunoglobulin (HBIG), and $18.8 \%$ did not know that newborn with HBsAg-positive mother could be breastfed after giving HBIG and vaccine. About 20.6\% of junior doctors did not know the possible withdrawal indications recommended by the guideline. For HBeAg-positive patients receiving low genetic barrier drugs, about $30 \%$ of junior doctors did not know that the antiviral strategies should be adjusted according to HBV DNA levels at treatment week 24. For CHB patients receiving chemotherapy, about $25 \%$ of participants did not know antiviral therapy should be started at least 1 week earlier, and about $20 \%$ did not know that adefovir dipivoxil or tenofovir disoproxil fumarate should be avoided in patients with kidney diseases or high-risk of developing kidney diseases.

Conclusions: The knowledge on CHB guideline was rather unsatisfactory in junior doctors. Our finding highlights the urgent need for strengthening junior doctors to gain a greater understanding of $\mathrm{CHB}$ guideline.
\end{abstract}

Keywords: Questionnaire, Knowledge, Chronic hepatitis B, Guideline, Junior doctor

\section{Background}

Hepatitis B virus (HBV) infection is a major health issue. Approximately two billion people worldwide are infected with HBV and about 240 million suffer from chronic hepatitis $\mathrm{B}(\mathrm{CHB})(1)$. In past decades, about $25 \%$ of adults who were infected with HBV during childhood died of decompensated liver cirrhosis or hepatocellular carcinoma (HCC) (1). However, due to the licensing of oral nucleos(t)ide, the treatment of CHB has been revolutionized with a significant improvement in disease progression and HCC occurrence $(2,3)$.

An epidemic survey published in 2006 estimated that 7.18\% of Chinese population were still hepatitis B surface antigen (HBsAg)-positive (4). Therefore, China is still considered to be a highly endemic region. Due to a relative lack of professional health services and patients' poor awareness and knowledge of disease, many CHB patients (especially poor patients living in rural areas) missed the opportunity for timely diagnosis and appropriate antiviral ther- apy. To standardize the diagnosis, treatment, and management of $\mathrm{CHB}$, the updated treatment guideline of hepatitis B (2015) has been formulated by the Chinese society of infectious diseases and Chinese society of hepatology, Chinese medical association (5). Afterwards, free continuing education courses of guide-interpretation have also been provided to clinicians who are engaged in the field of CHB.

\section{Objectives}

In real-life clinical practice, the diagnosis and treatment options of diseases are largely determined by the physicians; so, the level of primary physician's knowledge, training, and practice is closely related to the quality of medical services (6). Thus, this questionnaire-based survey was designed to assess the knowledge of junior doctors on the 2015 CHB guideline recommended by Chinese society of infectious diseases and Chinese society of hepatology, Chinese medical association. 


\section{Methods}

This was a cross-sectional study. The junior doctors working in Sichuan province of China in the field of $\mathrm{CHB}$ were invited to complete a questionnaire-based survey between May and July 2016. The questionnaire was validated by the scientific committee of the West China workgroup for the management of liver diseases.

In May 2016, this questionnaire was sent to all junior doctors who participated in a clinical training course hosted by our department. This survey was then extended to other junior doctors belonging to West China workgroup for the management of liver diseases (including 30 primary hospitals in 21 cities of Sichuan province, (see Figure 1). The questionnaires were sent and collected by post. In this survey, responses were collected before 31 July 2016.

The content of the questionnaire was based on the Chinese CHB guideline launched in 2015 (5), which consisted of 28 questions focusing on the following three aspects: mother-to-child transmission (MTCT) prevention, response-guided therapy (RGT) strategies, and special patients antiviral therapy (Table 1). The answer to each question was classified as known or unknown conforming to the recommendations from guideline. In this study, the questionnaire was available in simplified Chinese, and the draft questionnaire was revised by the research team through consensus.

\section{Results}

\subsection{General Characteristics of Participants}

A total of 600 junior doctors were invited to participate in this study. However, usable responses were received from 562 out of the 600 junior doctors, yielding a participation rate of $93.7 \%$. In those junior doctors, the mean age was 31.5 years; 329 ( $58.5 \%$ ) of them were male and 233 (41.5\%) were female; and $91.8 \%$ (516/562) of them were from infectious (contagious) diseases department.

\subsection{Responses on Infant Vaccination}

For the questions related to hepatitis B vaccine and hepatitis B immunoglobulin (HBIG) application in newborns exposed to HBsAg-positive mothers, $18.3 \%$ of junior doctors did not know the dose of HBIG ( $\geq 100 \mathrm{IU}$ ), $13.9 \%$ did not know that HBIG and vaccine should be given intramuscularly at different sites, and up to $32.1 \%$ of junior doctors did not know the type and dose of hepatitis B vaccine available in clinical practice.

For infants with weak immune system or low vaccine response, $24.9 \%$ of junior doctors did not know that the dose and frequency of vaccination could be increased. Additionally, $18.8 \%$ of respondents did not know that the newborns could be breastfed after giving HBIG and vaccine, if they were exposed to HBsAg-positive mothers.

\subsection{Responses on Response-Guided-Therapy Strategy}

In this study, about $20.6 \%$ of junior doctors did not know the possible withdrawal indications recommended by the guideline. For HBeAg-positive patients receiving lamivudine (LAM), telbivudine (LdT), or adefovir dipivoxil (ADV), 29.7\% of junior doctors did not know that the antiviral strategies should be adjusted according to HBV DNA levels at treatment week 24 .

For patients receiving interferon treatment, $29.7 \%$ of junior doctors did not know that the treatment should be stopped if serum HBsAg level was still more than 20,000 IU/mL after 24 weeks of treatment; and up to $35.3 \%$ did not know that the treatment should be stopped if there are no decline of serum HBsAg level and less than $2 \log \mathrm{IU} / \mathrm{mL}$ decline of HBV DNA.

\subsection{Responses Relating to Antiviral Therapy in Special Popula- tions}

For those patients with chemotherapy or immunosuppressive therapy, 6.4\% of junior doctors did not know that serum HBsAg, anti-HBc, and HBV DNA should be routinely screened; $23.9 \%$ did not know that antiviral therapy should be initiated at least one week earlier; and up to $43.1 \%$ did not know that prophylactic antivirus therapy should be considered for HBsAg-negative and anti-HBc-positive patients with immunotherapy using monoclonal antibodies to specifically target B cells.

For liver failure patients, $29.1 \%$ did not know that nucleos(t)ide analog (NAs) should be used for those with positive HBsAg but negative HBV DNA. For patients with high risk of HBV reinfection after liver transplantation, up to $34.3 \%$ of junior doctors did not know that low-dose HBIG plus NAs therapy should be the major antiviral strategy. For patients with negative-HBV DNA before liver transplantation and low risk of HBV reinfection after liver transplantation, up to $43.7 \%$ of junior doctors did not know that HBIG could not be used.

For active hepatitis B patients during pregnancy, 19.5\% of junior doctors did not know that tenofovir disoproxil fumarate (TDF) or LdT could be considered after obtaining the informed consent of the patients. For accidental pregnancy during antiviral therapy, $15.2 \%$ of participants did not know that pregnancy should be terminated during interferon treatment; and 19.2\% did not know that pregnancy could be continued during TDF or LdT treatment. For HBsAg-positive mothers with an HBV DNA level of more 


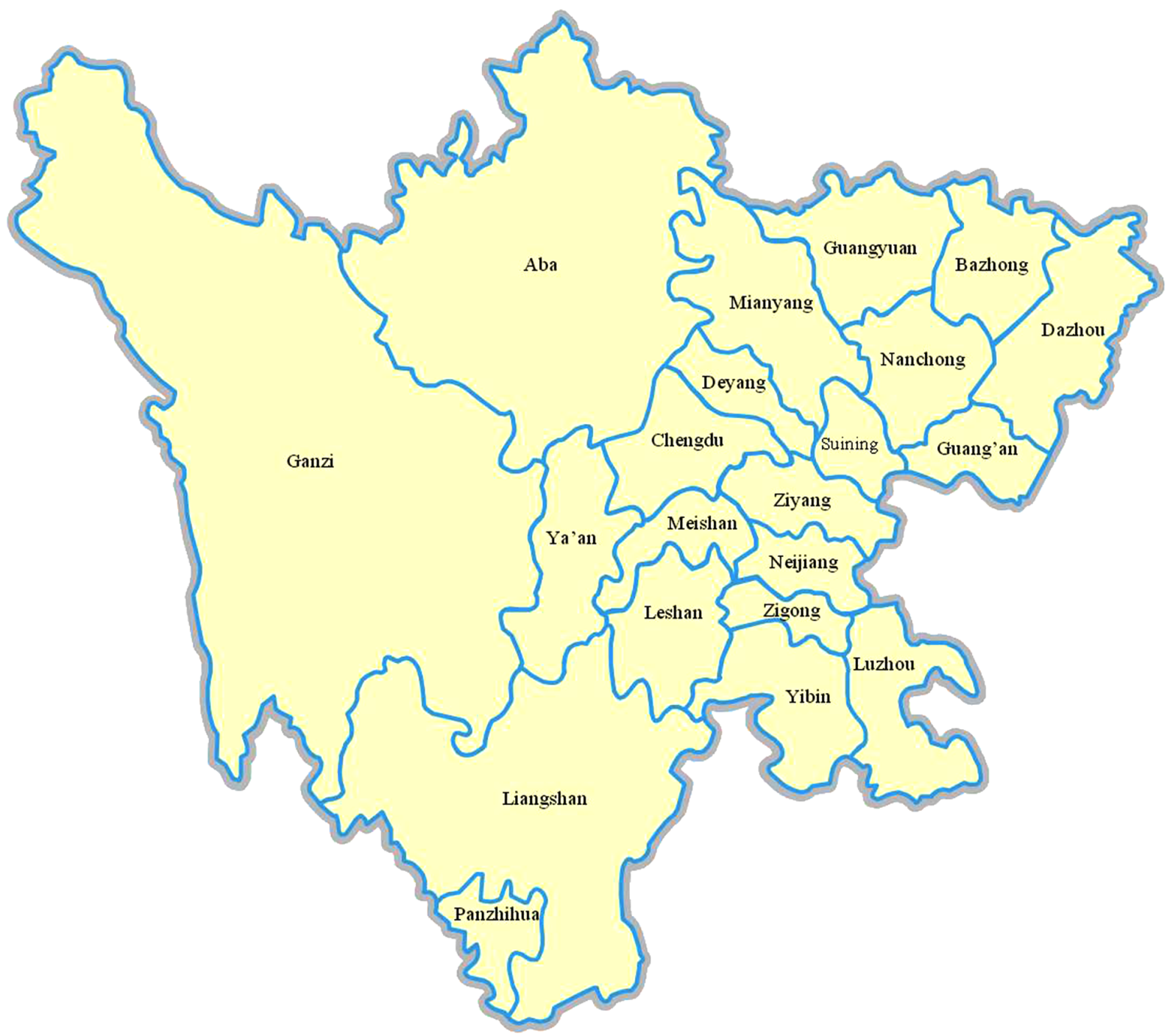

Figure 1. The Location of 30 Primary Hospitals in 21 Cities of Sichuan Province, China

than 2,000,000 IU per milliliter, up to $32.7 \%$ of junior doctors did not know that TDF and LdT could be used during the third trimester to prevent mother-to-child transmission.

Up to $30 \%-40 \%$ of junior doctors were not familiar with the antiviral related issues among children with progressive liver disease and cirrhosis. For patients with preexisting renal diseases or at high risk of renal diseases, 22.4\% of junior doctors did not know that ADV or TDF should be avoided as much as possible, and $25.5 \%$ of junior doctors did not know ETV or LdT could be considered.

\section{Discussion}

Hepatitis B is one of the most common infectious diseases in China, and it has greatly affected the physical health of the Chinese people as well as the economic and social development of the country (7). At present, improving the scientific understanding of hepatitis B for general population and sharing the professional medical knowledge encoded in a specific guideline for clinicians and other health-care providers are both extremely important for the effective prevention and treatment of $\mathrm{CHB}$ in real life clinical practice.

Previous studies have already revealed a lower knowledge on hepatitis B in general population, medical stu- 
dents, and patients (8-11). One study from China also investigated Chinese physicians' awareness regarding the 2010 guidelines on the treatment of CHB. The results showed that more than $10 \%$ of physicians did not adhere to the guidelines, and physicians from infectious disease or liver disease departments were better informed than those from gastrointestinal or other departments (12). To investigate the knowledge about the 2015 CHB guideline among the junior doctors from primary hospitals, several typical questions focusing on MTCT prevention, RGT strategies, and special patients antiviral therapy were surveyed. We found that about $30 \%$ of junior doctors were not familiar with the vaccine and HBIG used for infants with HBsAgpositive mother; about 20\% were unclear that the antiviral strategies should be adjusted according to the treatment responses; and about 20\% remained unaware of drug selection in CHB patients with kidney diseases or high risk of developing kidney diseases. To some extent, the results of our survey showed that the knowledge of "professional" clinicians on $\mathrm{CHB}$ guideline was unsatisfactory in real-life clinical practice.

In China, the specialist training in liver disease was just started in recent years, and the cultivation of specialists was quite casual and there were lack of unified standards in past decades $(13,14)$. Because of the limited resource of continuing education and low learning enthusiasm of health care providers, majority of them were not timely updated. This unfortunate situation was extremely common in regions with relatively backward economic and social development in China $(15,16)$. Indeed, the depth of continuing education directly influenced the quality of health care provided by specialists, and not participating in continuing education always had a negative effect on promoting liver health and quality of patient care.

In the present study, all junior doctors were engaged in the field of liver diseases; more than $90 \%$ of junior doctors had been working for more than 5 years, and all of junior doctors were from the second-grade of class-A hospitals or above. However, for each question listed in the questionnaire, nearly $10 \%$ 40\% of participants did not know the answer. Thus, for those who have already worked in the field of liver diseases in China and without professional training before work, it is still important to consider the accessibility of continuing education opportunities and awareness of junior doctors about the importance of continuing education. For those who are willing to be specialist physicians, the specialist training (including academic and clinical skills) in liver diseases must be received prior to working alone with patients in the field. To ensure the real implementation of continuing education and specialist training, there are lots of work that cannot be done without the supports from health authorities, Chi- nese medical association, and Chinese medical doctor association.

In summary, this study revealed an unsatisfactory level of knowledge on Chinese $\mathrm{CHB}$ guideline among junior doctors in primary hospitals of Sichuan province, China. Though there may be limitations on the sample size and representativeness of participants, the finding still highlights the urgent need for strengthening junior doctors to gain a greater understanding of the CHB guideline.

\section{Acknowledgments}

We thank all participants for their participation and all the staff of the survey team for their efforts that made this study possible. This work was supported by grants from the Science and Technology Support Program of Sichuan province, China (2015SZ0049).

\section{Footnote}

Authors' Contribution: Conceptualization of the study was done by En-Qiang Chen and Hong Tang. Methodology was designed by En-Qiang Chen, Hong Tang and Meng-Lan Wang. Original draft was written by En-Qiang Chen. Review and editing were performed by Hong Tang. Data were provided and analyzed by Lang Bai, Xiao-Fang Lv, Dong Mei Zhang, Juan Wang and Ya-Chao Tao. Supervision belonged to Hong Tang. En-Qiang Chen and Meng-Lan Wang contributed equally to this paper.

\section{References}

1. Ganem D, Prince AM. Hepatitis B virus infection-natural history and clinical consequences. $N$ Engl J Med. 2004;350(11):1118-29. doi: 10.1056/NEJMra031087. [PubMed: 15014185].

2. Rapti I, Hadziyannis S. Risk for hepatocellular carcinoma in the course of chronic hepatitis B virus infection and the protective effect of therapy with nucleos(t)ide analogues. World $J$ Hepatol 2015;7(8):1064-73. doi: 10.4254/wjh.v7.i8.1064. [PubMed: 26052395].

3. Su TH, Hu TH, Chen CY, Huang YH, Chuang WL, Lin CC, et al. Four-year entecavir therapy reduces hepatocellular carcinoma, cirrhotic events and mortality in chronic hepatitis B patients. Liver Int. 2016;36(12):1755-64. doi: 10.1111/liv.13253. [PubMed: 27634134].

4. Cui Y, Jia J. Update on epidemiology of hepatitis B and C in China. J Gastroenterol Hepatol. 2013;28 Suppl 1:7-10. doi: 10.1111/jgh.12220. [PubMed: 23855289].

5. Chinese Society of Hepatology CMA, Chinese Society of Infectious Diseases CMA, Hou JL, lai W. [The guideline of prevention and treatment for chronic hepatitis B: a 2015 update]. Zhonghua Gan Zang Bing Za Zhi. 2015;23(12):888-905. doi: 10.3760/cma.j.issn.10073418.2015.12.002. [PubMed: 26739464].

6. Mukhtar NA, Kathpalia P, Hilton JF, Lau G, Yu A, Grumbach K, et al. Provider, Patient, and Practice Factors Shape Hepatitis B Prevention and Management by Primary Care Providers. JClin Gastroenterol. 2016 doi: 10.1097/MCG.0000000000000738. [PubMed: 27811627]. 
Table 1. The Questions Listed in the Questionnaire in this Survey

\begin{tabular}{|c|c|}
\hline Section & Subject / Question \\
\hline Section 1 & Mother-to-Child Transmission (MTCT) Prevention \\
\hline Q1 & $\begin{array}{l}\text { Do you know that the dose of HBIG should be } \geq 100 \text { IU for } \\
\text { newborns exposed to HBsAg-positive mothers? }\end{array}$ \\
\hline Q2 & $\begin{array}{l}\text { Do you know that HBIG and vaccine should be given } \\
\text { intramuscularly at different sites? }\end{array}$ \\
\hline Q3 & $\begin{array}{l}\text { Do you know the type and dose of hepatitis B vaccine available } \\
\text { in clinical practice? }\end{array}$ \\
\hline Q4 & $\begin{array}{l}\text { Whether the dose and times of vaccination should be } \\
\text { increased for infants with weak immune system or low vaccine } \\
\text { response? }\end{array}$ \\
\hline Q5 & $\begin{array}{l}\text { If newborns were exposed to HBsAg-positive mothers, whether } \\
\text { they could be breastfed after giving HBIG and vaccine? }\end{array}$ \\
\hline Section 2 & Response-guided therapy (RGT) strategies \\
\hline Q6 & $\begin{array}{l}\text { Do you know the suggestions for treatment withdrawal } \\
\text { among HBeAg-positive patients? }\end{array}$ \\
\hline Q7 & $\begin{array}{l}\text { Do you know the time of consolidate treatment to patients } \\
\text { after HBeAg/anti-HBe seroconversion? }\end{array}$ \\
\hline Q8 & $\begin{array}{l}\text { For HBeAg-positive patients receiving lamivudine, telbivudine } \\
\text { or adefovir, whether the antiviral strategies should be adjusted } \\
\text { according to HBV DNA levels at treatment week } 24 \text { ? }\end{array}$ \\
\hline Q9 & $\begin{array}{l}\text { Whether the treatment should be stopped for patients } \\
\text { receiving interferon treatment, if serum HBsAg level was still } \\
\text { more than } 20,000 \mathrm{IU} / \mathrm{mL} \text { after } 24 \text { weeks of treatment? }\end{array}$ \\
\hline Q10 & $\begin{array}{l}\text { Whether pegIFN treatment should be stopped if there were no } \\
\text { decline in serum HBsAg level and less than } 2 \log \mathrm{IU} / \mathrm{ml} \text { decline } \\
\text { in HBV DNA? }\end{array}$ \\
\hline Section 3 & Special patients antiviral therapy \\
\hline Q11 & $\begin{array}{l}\text { Is pegIFN treatment absolutely forbidden for } \mathrm{CHB} \text { patients } \\
\text { with compensated cirrhosis? }\end{array}$ \\
\hline
\end{tabular}

Q12 Whether serum HBsAg, anti-HBc, and HBV DNA should be routinely screened in patients with chemotherapy or immunosuppressive therapy?

Q13 Do you know that antiviral therapy should be initiated at least one week before chemotherapy or immunosuppressive therapy?

Q14 Do you know that prophylactic antivirus therapy should be considered for HBsAg-negative and anti-HBc-positive patients with immunotherapy using monoclonal antibodies to specifically target B cells?

Q15 For liver failure patients, whether nucleos(t)ide analog should be used for those with positive HBsAg but negative HBV DNA?

Q16 Whether nucleos $(\mathrm{t})$ ide analog should be used for HCC patients with detectable HBV DNA?

Q17 The antiviral strategy for HBV-HIV co-infections with CD4+T cell $\leq 500 / \mathrm{mL}$

Q18 Do you know the major antiviral strategy for patients with high risk of HBV reinfection after liver transplantation?

Q19 Whether HBIG could be used for patients with negative-HBV DNA before liver transplantation and low risk of HBV reinfection after liver transplantation?

Q20 For active hepatitis B patients during pregnancy, which nucleos(t)ide analog could be considered after obtaining the informed consent of the patients?

Q21 For accidental pregnancy during antiviral therapy, whether pregnancy should be terminated during pegIFN treatment?

Q22 How should be dealt with pregnancy during TDF or LdT

Hepat Mon. 261\%, 19(3):e58695.

Q23 For HBsAg-positive mothers with HBV DNA level of more than 2,000,000 IU per milliliter, when should TDF and LdT treatment be given to prevent mother-to-child transmission?

Q24 Whether IFN could be considered for children with advanced liver disease or cirrhosis?
7. Jia J. A different look at the management of chronic hepatitis B in a resource-constrained country. J Viral Hepat. 2013;20 Suppl 1:1. doi: 10.1111/jvh.12056. [PubMed: 23458517].

8. Tamayo A, Shah SR, Bhatia S, Chowdhury A, Rao PN, Dinh P, et al. Correlates of disease-specific knowledge among patients with chronic hepatitis B or hepatitis C infection in India. Hepatol Int. 2016;10(6):988-95. doi: 10.1007/s12072-016-9728-3. [PubMed: 27146692].

9. Adjei CA, Asamoah R, Atibila F, Ti-Enkawol GN, Ansah-Nyarko M. Mother-to-child transmission of hepatitis B: extent of knowledge of physicians and midwives in Eastern region of Ghana. BMC Public Health. 2016;16:537. doi: 10.1186/s12889-016-3215-6. [PubMed: 27401399].

10. Ghomraoui FA, Alfaqeeh FA, Algadheeb AS, Al-Alsheikh AS, AlHamoudi WK, Alswat KA. Medical students' awareness of and compliance with the hepatitis B vaccine in a tertiary care academic hospital: An epidemiological study. J Infect Public Health. 2016;9(1):60-5. doi: 10.1016/j.jiph.2015.06.008. [PubMed: 26185014].

11. Tatsilong HO, Noubiap JJ, Nansseu JR, Aminde LN, Bigna JJ, Ndze VN, et al. Hepatitis B infection awareness, vaccine perceptions and uptake, and serological profile of a group of health care workers in Yaounde, Cameroon. BMC Public Health. 2016;15:706. doi: 10.1186/s12889-0163388-z. [PubMed: 27487845]

12. Wei L, Jia JD, Weng XH, Dou XG, Jiang JJ, Tang H, et al. Treating chronic hepatitis B virus: Chinese physicians' awareness of the 2010 guidelines. World J Hepatol. 2016;8(18):762-9. doi: 10.4254/wjh.v8.i18.762. [PubMed: 27366303].

13. Zhu J, Li W, Chen L. Doctors in China: improving quality through modernisation of residency education. Lancet. 2016;388(10054):19229. doi:10.1016/S0140-6736(16)00582-1. [PubMed: 27339756].

14. Li D. Training doctors for primary care in China: Transformation of general practice education. J Family Med Prim Care. 2016;5(1):1-2. doi: 10.4103/2249-4863.184614. [PubMed: 27453834].

15. Wang HH, Wang JJ, Zhou ZH, Wang XW, Xu L. General practice education and training in southern China: recent development and ongoing challenges under the health care reform. Malays Fam Physician. 2013;8(3):2-10. [PubMed: 25893051].

16. Wong WC, Jiang S, Ong J, Peng M, Wan E, Zhu Z, et al. Availability and use of primary care facilities in China: a nationwide representative survey. Lancet. 2016;388 Suppl 1:S18. doi: 10.1016/S0140-6736(16)319456. [PubMed: 27968830]. 